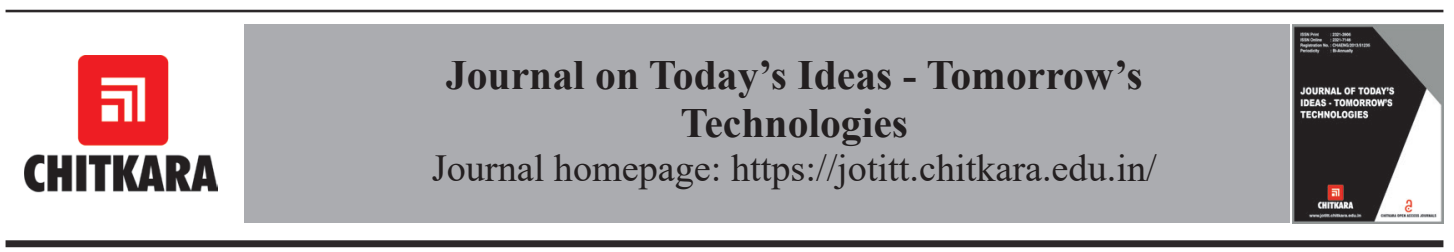

\title{
Semi-Automatic Sanitizer and Thermal Scanner
}

\author{
Jasmeet Singh*, Gurmeet Singh and Gurditt Singh \\ Chitkara University Institute of Engineering \& Technology, Chitkara University, Punjab-140401, India
}

Email: myselfjasmeet@gmail.com*

\section{ARTICLE INFORMATION}

Received: June 14, 2020

Revised: June 24, 2020

Accepted: June 27, 2020

Published online: December 03, 2020

Keywords:

COVID-19, Semi-Automatic sanitizer,

Pandemics, Thermal scanner

\begin{abstract}
War of humanity against COVID-19 is stretching at this stage of time. In this paper, a solution has been proposed to sanitize the narrow roads and public areas with a robotic solution. Robot design proposed in this paper can be operated using a remote and that saves human beings from direct possible face off with COVID-19 virus.. This Semi-Automatic machine proposed in the work has a built-in thermal scanner to scan the locals and also a walkie talkie to give further instructions to the person being scanned.Robot also has a carriage section that can transport medicine and food to the infected area. With this one robot, a large area such as slums, narrow roads, schools, and buildings etc. can be covered.
\end{abstract}

DOI: $10.15415 /$ jotitt.2020.81007

\section{Introduction}

The emergence of Severe Acute Respiratory Syndrome Coronavirus 2 (SARS-CoV-2) in China in December 2019 had led to a global outbreak of coronavirus disease 2019 (COVID-19). Disease soon started to spread all over the world and became an international public health issue. The entire humanity has to fight in this war against the unexpected coronavirus and each and every individual has to play an important role for possible curb of the issue. The healthcare system is doing exceptional work and the government is taking various measures that can help the society to control the spread. Strategies utilizing potential technologies would yield better benefits and these technological strategies can be framed either to control the pandemic or to support the confinement of the society during pandemic which in turn aids in controlling the spreading of the infection [1].
The current scenario has motivated the experts to find a cure for the disease, identify frequently changing presentation of the symptoms, provide efficacy in the treatment and overcome the tedious rehabilitation of patients. Furthermore, the researchers have shown a keen interest in providing public support in the form of sanitization for the prevention of disease spread but have failed to provide safety of staff, costeffective models and services at areas that are not easily reachable. The main motive of this study is to widen the spectrum of public health facilities by providing support at ground level i.e accessibility of medical, food and sanitation services in areas having narrow and sleek passages while keeping in mind the no-contact policy.

\subsection{Agents Used to Sanitize the Areas}

Inactivation of corona viruses by biocidal agents in suspension tests Ethanol (78-95\%), 2-propanol 
(70-100\%), the combination of 45\% 2-propanol with $30 \%$ 1-propanol, glutardialdehyde (0.5$2.5 \%)$, formaldehyde $(0.7-1 \%)$ and povidone iodine $(0.23-7.5 \%)$ readily inactivated coronavirus infectivity by approximately $4 \log _{10}$ or more. Sodium hypochlorite found to be required a minimal concentration of at least $0.21 \%$ to be effective. Hydrogen peroxide was effective with a concentration of $0.5 \%$ and an incubation time of $1 \min [2]$.

In non-health care settings, sodium hypochlorite (bleach) may be used at a recommended concentration of $0.1 \%$ (1000 ppm). Alternatively, alcohol with $70 \%-90 \%$ concentration may be used for surface disinfection [3].

\subsection{Already Available Similar Solutions/ Products}

There are already available similar products fighting with COVID-19 by providing different features and facilities to sanitize the public areas. Some of the equipments that are already available are drones, sports equipments sanitizing device, pressurized sanitizer box and tanks.

Drones: According to the recent interventions amid the pandemic the most interesting one is the use of drones for medical, parcel, and grocery deliveries, monitoring the situations as well as spraying disinfectants. Various drones that are available are delivery drones, Inspection, broadcasting and Crowd Control drones, Spraying Disinfectants drone, Patient Monitoring drones. All the drones have different features and ways to tackle the spread of COVID-19 [4].

Sanitizing sports equipment: An apparatus is provided for sanitizing sports equipment which comprises of a cabinet thatcan be sealed,. There are first and second conduits in fluid communication with the cabinet, an ozone generator and fans for circulating ozone containing gas through the conduits into the cabinet. Fans are being operative to circulate the ozone containing gas in a first direction and subsequently in a reverse direction. There is a provision for fluid storage device being associated with a spray action to spray the fluid into the cabinet after the ozone has been circulated [5].

Tru-D Rapid Room Disinfection: Environmental surfaces play an important role in transmission of healthcare associated pathogens. The Tru-D Rapid Room Disinfection device is a mobile, fully-automated room decontamination technology that utilizes ultraviolet-C radiations to kill pathogens. Disinfection of hospital rooms with Tru-D reduced the frequency of positive methicillin-resistant Staphylococcus aureus (MRSA) and vancomycin-resistant Enterococcus (VRE) cultures by $93 \%$ and of Clostridium difficile cultures by $80 \%$ [6].

Pressurized sanitizer box and tanks: Another technique that is used to sanitize the locality and other public areas is through pressurized sanitizer box which is carried on the back of the person sanitizing the locality and the spraying nozzle is in the hand of the person. In addition to this, big tanks can be used for large areas in which sanitizer is filled and sprayed out with the help of pipe. This technique requires 3-4 persons for sanitization of area.

Semi-Automatic sanitizer and thermal scanner is different from already available solutions as in this all the facilities like sanitizing box, thermal scanner, delivery box, camera and walkie talkie are available. This device can be used for the various purposes like sanitizing the public areas, delivery of medicine and food, scanning of the temperature of target society and supply medicines to patient bed in hospitals.. This device requires less number of persons to control the device and sanitize the area thus reducingthe chances of direct contact with other person and prevent inhalation of chemicals of sanitizer.

\section{Methodology}

Semi Automatic sanitizer and thermal scanner is a remote control machine that will have sanitizer, thermal scanner and box for supplying food and medicine as main function.

Sanitizer kit includes sanitizer storage tank, chassis and Pumping system through nozzles 
are present on the front of the device (Fig.1). Electronic System, High definition 360-degree camera and thermal camera are installed behind the sanitizer storage tank (Fig. 2). 3D view of the device is shown in Fig. 3(a) and $3(\mathrm{~b})$ for a complete viewpoint.

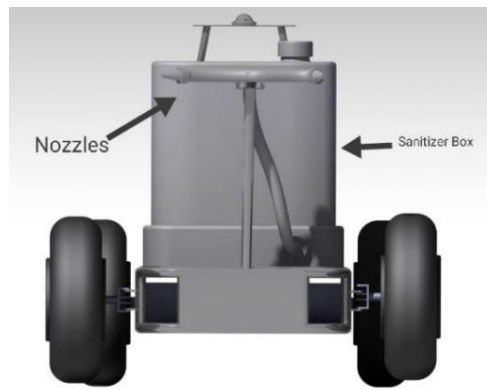

Figure 1: Front view of Semi Automatic Sanitizer and Thermal Scanner

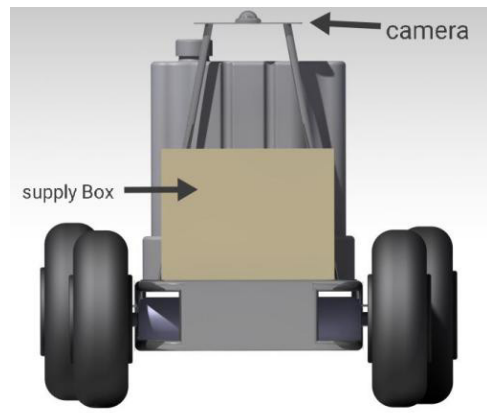

Figure 2: Back view of Semi Automatic Sanitizer and Thermal Scanner

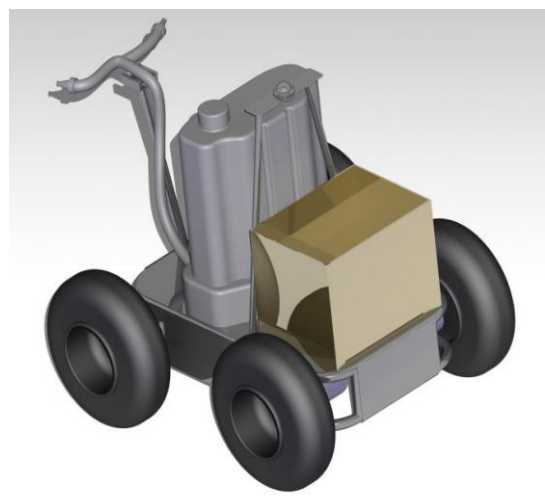

(a)

\section{Construction}

(i) Chassis - The Pipe material used in the chassis is Carbon Steel AISI 1020 with dimension of Outer diameter 1 inch and thickness $2 \mathrm{~mm}$. An Aluminium sheet is used as body panel. The dimension and the materials are decided by carrying out the FEA Analysis on Simscale. Total deformation and safety factors are shown in Fig. 4 and Fig. 5 respectively.

(ii) Electronics System - Electronic System includes different electronics components such as Mega Arduino, Radio Frequency Module, two $12 \mathrm{~V}$ Motor to drive the rear wheels, one Stepper motor to steer front tyres and motor drivers for all the motors.

(iii) Suspension and Steering System - The suspension parameters are as follows with rear track width of $820 \mathrm{~mm}$ and front track width of $700 \mathrm{~mm}$ and a wheelbase of $575 \mathrm{~mm}$. A simple steering system is used in which the steering arms slides by a pivot which is controlled by stepper motor. These types of the steering system are used in LCVs.

(iv) Sanitization System - A sanitizing system includes a sanitizing storage tank with capacity of $40 \mathrm{~L}$ and a semiautomatic reciprocating pumping system powered by a DC $12 \mathrm{~V}$ motor and nozzle tips are provided for pressure spraying.

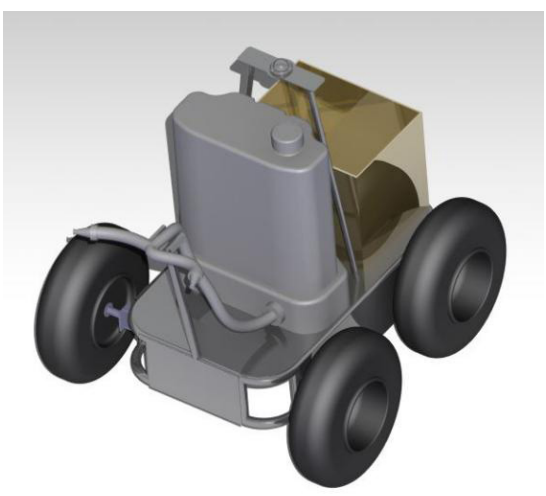

(b)

Figure 3: 3D view of Semi Automatic Sanitizer and Thermal Scanner (a) left side view and (b) right side view 


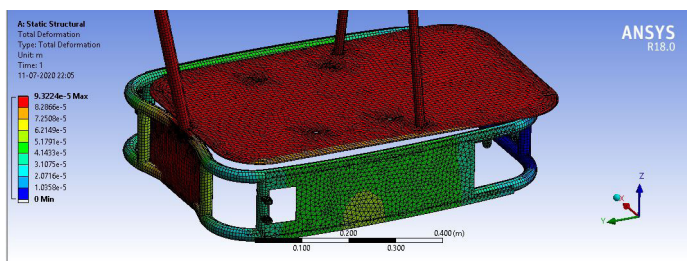

Figure 4: Total deformation factors of Semi Automatic Sanitization Machine

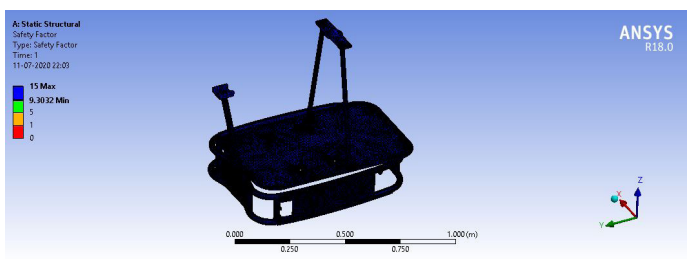

Figure 5: Safety factor of Semi Automatic Sanitization Machine

The working of the device for the three different places - narrow roads, hospitals and public areas are as follows.

The person controls the device through remote which can stay outside the narrow roads and send the device inside the narrow roads with the help of a camera installed on the top for navigation. The range of the device is about $1 \mathrm{~km}$. The camera will also help to record the areas covered and check a person's temperature through thermal sensor. When the device will go inside the narrow locality, an announcement will be done to alert the people before sanitizing the locality with the help of a walkie talkie that is installed in the device.

Semi-Automatic sanitizer controls are provided on remote that will help to sanitize the narrow roads. Nozzles are provided right and left side to cover more area. High pressure pumping system is installed inside sanitizer box which pumps the sanitizing substance at pressure. With the help of nozzle tips the sanitizer is sprayed. To check the temperature of any person from this narrow locality, thermal scanner can easily detect it from a distance of 5 meter and the reading of the temperature will be displayed on the remote. Medicine and food can be put inside the box to supply it to the sealed areas without entering the area. To prevent any damage to the device, the pressure horn will blow if any animal or human try to harm the device.

In hospitals, the device can be used to supply the medicine and food to the patient bed. Blood and sputum samples can also be kept in the supply box that can be taken to the lab with the help of the device. Hospital requires every minute sanitization to prevent the spread of disease. The sanitization of the hospital can be easily done by this machine.

In various public areas like Malls, schools, colleges, parks etc. the device can be used for continuous sanitization of the area and thermal screening of persons entering these places.

\section{Results and Discussion}

This Semi-Automatic sanitizer and thermal scanner is effective in sanitizing narrow roads effectively. Normal speed of this device is $20 \mathrm{Km} / \mathrm{h}$, during sanitization, the device will be made to move at a speed of $5 \mathrm{~km} / \mathrm{h}$ for better results. So to sanitize a road of 100 meter length, it will take 2.28 minutes. As a whole, this device will help to a great extent in sanitizing each and every place easily and quickly. Even the government can also use this device to sanitize public areas and control this pandemic by breaking the chain of spread. As this device is a full pack of benefits and features so it will be of great benefit for everyone.

Still, there is a scope of enhancement and changes that will be done later on. As we will be adding Artificial Intelligence in it to make this device more effective and worthy.

\section{Conclusion}

This device is a sanitizer kit which can sanitize the whole locality without requiring a human to enter the infected locality as it can be navigated from a distance by using front camera on it. It also contains a thermal scanner and a walkie talkie for giving additional commands to the person being inspected. It also contains additional box for medicine and food supply and it can be used in highly infected locality with narrow roads, schools, 
malls and other public area. At last we conclude that this device is capable of covering large areas.

\section{References}

[1] R. M. Elavarasan \& R. Pugazhendhib. Restructed society and environment: A review on potential technological strategies to control the COVID - 19 pandemic. Science of the Total Environment, 725, 2020. https://doi.org/10.1016/j scitotenv.2020.1 38858

[Q] G. Kampf, D. Todt, S. Pfaender, E. Steinmann. Persistence of coronaviruses on inanimate surfaces and their inactivation with biocidal agents. Journal of Hospital Infection, vol. 104, no. 3, pp. 246-251. https://doi.org/10.1016/j.jhin.2020.01.022

[3] WHO. Cleaning and disinfection of environmental surfaces in the context of COVID-19: world health organization. [Online] Available: https://www.who. int/publications/i/item/cleaning-anddisinfection-of-environmental-surfacesinthe-context-of-covid-19] [Accessed 15 May 2020].

[4] P. Singla. Drone Technology - Game Changer to Fight Against COVID-19. Tathapi, vol. 19, no. 6, 2020.

[5] S. Steve. Sanitizing cabinet for sports equipment. Patent no. US20050193585A1, 2005.

[6] M. N. Michelle, L. C. Jennifer, J. P. Michael, J. D. Curtis. Evaluation of an automated ultraviolet radiation device for decontamination of Clostridium difficile and other healthcare-associated pathogens in hospital rooms. BMC Infect Dis, pp. 10$197,2010$.

https://doi.org/10.1186/1471-2334-10-197

This article has been published as a part of Special regular issue of Journal on Today's Ideas Tomorrow's Technologies. Most of the articles in this issue are the papers for which the original ideas were presented at NOVATE 2020 (https://www.chitkara.edu.in/novate), a competition organized by Chitkara University and supported by Ministry of Electronics and Information Technology, Govt. Of India, Institution's Innovation Council, NSTEDB, Govt. Of India and FICCI. Student participants have written most of these articles. Some basic editing work has been done at the editorial level, but same have not been corrected very rigorously so as to preserve basic touch of student's writing.
旬
CHITKARA
Journal on Today's Ideas - Tomorrow's Technologies
Chitkara University, Saraswati Kendra, SCO 160-161, Sector 9-C, Chandigarh, 160009, India

Volume -8, Issue-1

June 2020

ISSN 2321-3906

Copyright: [` 2020 Jasmeet Singh, Gurmeet Singh and Gurditt Singh] This is an Open Access article published in Journal on Today's Ideas - Tomorrow's Technologies by Chitkara University Publications. It is published with a Creative Commons Attribution- CC-BY 4.0 International License. This license permits unrestricted use, distribution, and reproduction in any medium, provided the original author and source are credited. 